\title{
Multi-area distributed three-phase state estimation for unbalanced active distribution networks
}

\author{
Sheng CHEN ${ }^{1}$, Zhinong WEI ${ }^{1}$, Guoqiang $\mathrm{SUN}^{1}$, Ning $\mathbf{L U}^{2}$, \\ Yonghui SUN ${ }^{1}$, Ying $\mathrm{ZHU}^{1}$
}

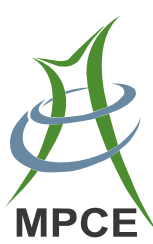

\begin{abstract}
This paper proposes a new multi-area framework for unbalanced active distribution network (ADN) state estimation. Firstly, an innovative three-phase distributed generator (DG) model is presented to take the asymmetric characteristics of DG three-phase outputs into consideration. Then a feasible method to set pseudo -measurements for unmonitored DGs is introduced. The states of DGs, together with the states of alternating current (AC) buses in ADNs, were estimated by using the weighted least squares (WLS) method. After that, the ADN was divided into several independent subareas. Based on the augmented Lagrangian method, this work proposes a fully distributed three-phase state estimator for the multi-area ADN. Finally, from the simulation results on the modified IEEE 123-bus system, the effectiveness and applicability of the proposed methodology have been investigated and discussed.
\end{abstract}

Keywords Active distribution networks (ADNs), Distributed three-phase state estimation, Three-phase distributed generator (DG) models, Pseudo-measurements, Augmented Lagrangian

CrossCheck date: 14 June 2016

Received: 3 March 2016/ Accepted: 28 June 2016/Published online: 4 November 2016

(C) The Author(s) 2016. This article is published with open access at Springerlink.com

$\triangle$ Zhinong WEI

wzn_nj@263.net

1 College of Energy and Electric Engineering, Hohai University, Nanjing 211100, China

2 Department of Electrical and Computer Engineering, North Carolina State University, Raleigh, NC 27695-7911, USA

\section{Introduction}

\subsection{Motivation}

The emergence and significant penetration of distributed generators (DGs) are transforming conventional passive distribution networks (PDNs) into active distribution networks (ADNs). Lots of new problems (e.g., overvoltage, reversed flows and overflows), however, have been brought into the distribution networks. These new challenges and the tendency moving towards smart distribution systems both require more comprehensive and accurate knowledge of the system states to implement real-time monitoring and active control for ADNs.

State estimation (SE) [1,2] provides a snapshot of network operating conditions from continuously updated measurements collected by supervisory control and data acquisition (SCADA) systems. SE has been a crucial tool for transmission system energy management systems (EMSs), where a variety of advanced applications for the economic, secure and reliable operation of power systems depend on the real-time states calculated by SEs. Analogously, for smart distribution systems, the quick and sharp fluctuations of DG outputs [3] and the frequent change of the network topology urge the distribution system operators to assess the operating conditions in an accurate and fast manner. Consequently, there is an increasing need for SEs that can enable enhanced grid situational awareness in the smart distribution system applications. To fulfill this target, three-phase SEs should be performed since distribution systems are inherently unbalanced with their unbalanced loads and network parameters [4].

\subsection{Literature review}

Previous works on conventional passive distribution network state estimation (PDNSE) can be summarized, 
roughly speaking, as three aspects: (1) The choice of state variables [5-7]. Apart from choosing voltage as state variables, branch-current based SEs were implemented to improve the computational efficiency and reduce the sensitivity to network parameters [5-7]; (2) The choice of state estimator [8-10]. In $[8,9]$, robust estimators were investigated to eliminate bad data out of redundant measurements. Gol and Abur [8] proposed a least absolute value (LAV) estimator for a three-phase SE. In [9], a new equivalent weight function was developed. Singh et al. [10] investigated the choice of state estimators for distribution systems, and concluded that weighted least squares (WLS) based SE was the most appropriate method because of the low redundancy levels. (3) Measurement configuration and observability issues [11-13]. Since distribution networks are short of sufficient metering infrastructures, load estimation $[9,11]$ based on prior knowledge of load variation pattern was carried out to function as pseudo -measurements. Meanwhile, depending heavily on the low-performance pseudo-measurements, the SE's quality is probably too low to ensure an effective network management and control. As regards this, considerable efforts have been devoted to determine the proper number and placement of measurement instruments to guarantee the network observability, minimum investments and required accuracy $[12,13]$.

In comparison with PDNSE, the major issue for active distribution network state estimation (ADNSE) lies in the modeling of DGs. A single-phase SE for distributed networks integrated with DGs was performed in [14]. In [15], the DG units were modeled as PQ bus injection, without any detailed models of DGs. In [16], sequence-based models for different types of DGs were proposed. Pseudomeasurements for the DG three-phase total outputs, obtained from historical database or weather forecast, were set for unmonitored DGs in [16, 17]. Moreover, the phase outputs of DGs were determined with phase-based averaging. However, this phase-based averaging approach degrades the SE accuracy because the phase outputs of DGs largely depend on the unbalanced operating conditions $[18,19]$. It is often assumed that the three-phase output powers of DGs are balanced. However, that is not always the case in light of the unbalanced interfacing conditions. To conclude, the asymmetric characteristics of distribution networks or DG three-phase outputs have not been well considered.

Another intractable challenge for ADNSE is that, due to the typically large scale of distribution networks, the unbalanced three-phase network structure and the extensive integration of DGs, significant computational efforts can be required [20]. Furthermore, the high resistance/resactance $(R / X)$ ratios of distribution network branches could lead to worrying numerical stability issues for
ADNSE. The SE numerical stability issue was well addressed by convex semidefinite programming proposed in [21], but with a considerable computation burden. Compared with integrated state estimation (ISE), multiarea distributed state estimation (DSE), in which the largescale network is divided into several subareas and the SE in each area is performed in parallel, shows a higher computational efficiency and a better numerical stability. Numerous studies have provided elegant foundations for DSE at the transmission level [22-27]. Interested readers can refer to [22] for state of the art of DSE methods in multi-area transmission systems. But the works dealing with distribution network DSE are somewhat limited [28-30]. In [28], a new two-step DSE procedure was performed in distribution networks with additional measurement devices to be shared among neighboring areas. Agent-based DSE methods were discussed in [29] and [30].

\subsection{Contribution and paper organization}

Based on the above discussion, this work proposes an innovative distributed three-phase state estimation method for unbalanced ADNs. First, detailed three-phase steadystate models of DGs have been presented with consideration towards the asymmetric characteristics of DG threephase outputs. Next, a feasible strategy to set pseudomeasurements for unmonitored DGs was employed. After that, we developed a multi-area DSE scheme based on the concept of extended sub-areas and the augmented Lagrangian relaxation method. Meanwhile, the information exchanged between adjacent areas has been interpreted as equivalent pseudo-measurements for extended boundary buses. The primary contributions of this paper can be summarized as:

1) By taking into account of unbalanced DG phase outputs, the accuracy of SE for ADNs integrated with multi-type DGs is ensured.

2) A fully distributed state estimator is proposed, in which only a very limited data is exchanged between neighboring areas and no central processor is needed.

3) Even though the states of DGs and extended boundary buses were added into state variables, the observability for DSE still holds on.

4) The DSE framework developed in this research can simulate large-scale unbalanced networks and produce a fast, accurate and reliable solution.

For the remainder of this paper, the WLS-based SE for PDNs model is introduced in Section 2. In Section 3, SE for unbalanced ADNs integrated with different types of DGs is presented. Section 4 describes the DSE formulations. After that, Section 5 presents test results and their interpretations. Finally, Section 6 draws the conclusions. 


\section{WLS-based SE for PDNs}

WLS provides the maximum likelihood estimation by solving the following optimization problem:

$\min _{\mathbf{x}} J(\boldsymbol{x})=(z-h(\boldsymbol{x}))^{\mathrm{T}} \boldsymbol{R}^{-1}(\boldsymbol{z}-h(\boldsymbol{x}))$

where $z \in \Re^{m}$ is the measurement vector ( $m$ denotes the number of measurements); $\boldsymbol{x} \in \Re^{n}$ denotes the state variable vector ( $n$ represents the number of buses); $R=\operatorname{diag}\left\{\sigma_{1}^{2}, \sigma_{2}^{2}, \cdots, \sigma_{m}^{2}\right\} ; \sigma$ is the standard deviation; $\boldsymbol{R}^{-1}$ is the weighted matrix; $h(\cdot)$ is a nonlinear function relating $\boldsymbol{x}$ and $z ; m \geq n$ is a necessary condition for an observable system in which the state variables $\boldsymbol{x}$ can be uniquely obtained from the measurements $z$.

In this work, three-phase complex voltages have been selected as state variables (the root node is taken as the slack node, and its voltage is constant):

$\boldsymbol{x}=\left[\begin{array}{ll}\boldsymbol{U}^{\mathrm{abc}} & \boldsymbol{\delta}^{\mathrm{abc}}\end{array}\right]^{\mathrm{T}}$

where $\boldsymbol{U}^{\mathrm{abc}}$ and $\boldsymbol{\delta}^{\mathrm{abc}}$ are the three-phase voltage magnitude and angle, respectively.

As for the measurements in distribution networks, virtual measurements at the tied nodes (zero injected power) and pseudo-measurements should be exclusively included considering the poor number of real-time measurements. Fortunately, promising developments in phasor measurement units (PMUs) [8] and smart meters [31] are expected to facilitate the SE at the distribution levels.

It is known that the WLS-based SE problem (1) is inherently a nonlinear optimization problem, to solve it the first-order optimality conditions should be satisfied at the beginning. Then, the Gauss-Newton method is used to linearize the first order optimality conditions. Finally, the iterative formulation of (1) can be expressed as:

$\left\{\begin{array}{l}\Delta \boldsymbol{x}^{(k)}=\left[\boldsymbol{H}^{\mathrm{T}}\left(\boldsymbol{x}^{(k)}\right) \boldsymbol{R}^{-1} \boldsymbol{H}\left(\boldsymbol{x}^{(k)}\right)\right]^{-1} \boldsymbol{H}^{\mathrm{T}}\left(\boldsymbol{x}^{(k)}\right) \boldsymbol{R}^{-1}\left(z-h\left(\boldsymbol{x}^{(k)}\right)\right) \\ \boldsymbol{x}^{(k+1)}=\boldsymbol{x}^{(k)}+\Delta \boldsymbol{x}^{(k)}\end{array}\right.$

where $k$ denotes the iteration index and $\boldsymbol{H}$ is the measurement Jacobian maxtrix.

\section{Three-phase SE for unbalanced ADNs}

A model considering the asymmetric characteristics of DG three-phase outputs is proposed to perform three-phase SE for unbalanced ADNs. Additionally, the operating states of DGs have been added into the state variables. Furthermore, a feasible strategy setting pseudo-measurements for unmonitored DGs is presented to guarantee the observability of ADNs.

\subsection{Modeling of DGs}

Motivations to present a detailed three-phase DG model include:

1) In engineering practice, the total three-phase power outputs are specified for each DG, but cannot be directly treated as SE measurements.

2) A situation, where the total three-phase power outputs of DGs are within their permissive limits but individual phase outputs are overloaded, could happen under unbalance operating conditions. As such, individual phase outputs of DGs should be accurately estimated when the total three-phase outputs are specified.

Generally, there are two ways for DGs connecting to ADNs (note that single-phase and two-phase DGs are not considered in this article):

1) DGs (e.g. the wind power and hydropower) are directly connected to ADNs through a synchronous or induction generator. The model of DGs directly connecting to ADNs is shown in Fig. 1.

2) DGs (e.g. solar PVs, fuel cells, doubly-fed induction generators (DFIGs), and storages) are integrated into ADNs through the pulse width modulation (PWM) voltage source converter (VSC) [32]. The model of DGs connecting to ADNs through PWM converters is shown in Fig. 2. The primary DG sources are not directly included in the model since it is assumed that DG outputs are perfectly controlled by PWM converters.

In Figs. 1 and 2, $E_{0}$ and $\delta_{0}$ are the voltage magnitude and angle of DGs (in Fig. 1) or PWM converters on the AC side (in Fig. 2); $P_{\text {in }}$ and $Q_{\text {in }}$ are the three-phase total active and reactive powers injected into the AC network; $P_{0}$ and $Q_{0}$ are the three-phase total active and reactive outputs of DGs (in Fig. 1) or PWM converters on the AC side (in Fig. 2); $P_{\mathrm{DC}}$ and $U_{\mathrm{DC}}$ are the power and voltage of PWM converters on the direct current (DC) side; $M_{0}$ is the

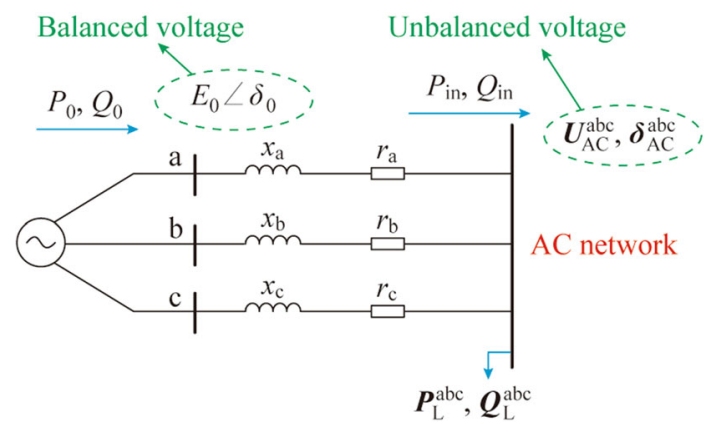

Fig. 1 Model of DGs connected to the grid directly 


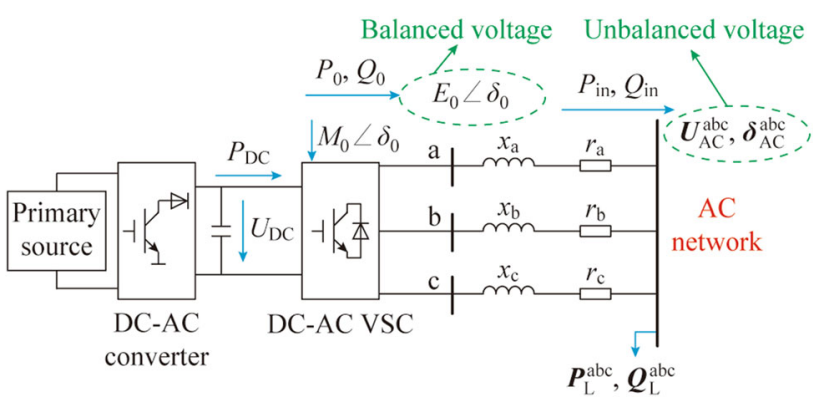

Fig. 2 Model of DGs connected to the grid through PWM

modulation coefficient of PWM converters; $\boldsymbol{P}_{\mathrm{L}}^{\mathrm{abc}}$ and $\boldsymbol{Q}_{\mathrm{L}}^{\mathrm{abc}}$ are the complex loads connected to the AC network; $\boldsymbol{U}_{\mathrm{AC}}^{\mathrm{abc}} \angle \boldsymbol{\delta}_{\mathrm{AC}}^{\mathrm{abc}}$ is the complex voltage of the AC bus to which the DG is connected; $r_{\varphi}(\varphi \in\{\mathrm{a}, \mathrm{b}, \mathrm{c}\})$ is the equivalent resistance for power loss; $\boldsymbol{x}_{\varphi}(\varphi \in\{\mathrm{a}, \mathrm{b}, \mathrm{c}\})$ is the equivalent reactance for the transformer and VSC filter.

It should be noted that nodal voltages of both directly connected DGs as presented in Fig. 1 (with balanced designs) and PWM converters as presented in Fig. 2 (with symmetric switching) are symmetric. As such, $E_{0}$ and $\delta_{0}$ are single-phase state variables. However, due to the unbalanced network parameters and loads, the states for the AC buses need to be represented by the three-phase state variables $\boldsymbol{U}^{\text {abc }}$ and $\boldsymbol{\delta}^{\text {abc }}$.

In accordance with the detailed DG models proposed here, the operating states of DGs are included in state variables as:

$\boldsymbol{x}_{\mathrm{AC}}=\left[\begin{array}{lll}\boldsymbol{x} & E_{0} & \delta_{0}\end{array}\right]^{\mathrm{T}}$

where $\boldsymbol{x}_{\mathrm{AC}}$ is the augmented state variable vector on the AC side.

With new state variables of DGs introduced, conventional measurements cannot guarantee the observability of ADNs because it is not realistic to equip all DGs with realtime metering devices. As a result, the system operators might lose the ability to be thoroughly aware of ADN operating conditions. Given this, it is of significant importance to effectively observe unmonitored DGs; this will be analyzed in the next subsection.

\subsection{Set pseudo-measurements for unmonitored DGs}

Let $\boldsymbol{z}_{\mathrm{DG}}$ denote the pseudo-measurements setting for unmonitored DGs. Then, the augmented measurements on the AC side become:

$\boldsymbol{z}_{\mathrm{AC}}=\left[\begin{array}{ll}\boldsymbol{z} & \boldsymbol{z}_{\mathrm{DG}}\end{array}\right]^{\mathrm{T}}$

where $z_{\mathrm{AC}}$ is the augmented measurement vector.

Since the augmented state variables $\boldsymbol{x}_{\mathrm{AC}}$ contain additional state variables $E_{0}$ and $\delta_{0}$, the dimension of the newly introduced measurements $z_{\text {DG }}$ needs to satisfy:
$\operatorname{dim}\left(z_{\mathrm{DG}}\right) \geq \operatorname{dim}\left(\left[E_{0} \delta_{0}\right]\right)$

In order to satisfy (6), one feasible and realistic strategy to set pseudo-measurements for the unmonitored DGs connecting to ADNs either directly or through PWM converters is illustrated as follows.

\subsubsection{DGs connected to ADNs directly}

The three-phase total active outputs $P_{0}$ of DGs can be estimated from historical database or forecasted weather data $[16,17]$. After that, according to $P_{0}$, the three-phase total reactive outputs $Q_{0}$ of DGs can be calculated from the $P-Q$ diagram or the power factor of DGs. Therefore, $P_{0}$ and $Q_{0}$ can be included in the pseudo-measurements $z_{\text {DG }}$ as:

$z_{\mathrm{DG}}=\left[\begin{array}{ll}P_{0} & Q_{0}\end{array}\right]$

The pseudo-measurements $z_{\mathrm{DG}}$ in (7) can be explicitly related to the augmented state variables $\boldsymbol{x}_{\mathrm{AC}}$ as follows:

$$
\begin{aligned}
P_{0}= & \sum_{\varphi \in\{\mathrm{a}, \mathrm{b}, \mathrm{c}\}} E_{0}^{2} g^{\varphi}-E_{0} U_{\mathrm{AC}}^{\varphi} g^{\varphi} \cos \left(\delta_{0}^{\varphi}-\delta_{\mathrm{AC}}^{\varphi}\right) \\
& -\sum_{\varphi \in\{\mathrm{a}, \mathrm{b}, \mathrm{c}\}} E_{0} U_{\mathrm{AC}}^{\varphi} b^{\varphi} \sin \left(\delta_{0}^{\varphi}-\delta_{\mathrm{AC}}^{\varphi}\right) \\
Q_{0}= & \sum_{\varphi \in\{\mathrm{a}, \mathrm{b}, \mathrm{c}\}}-E_{0}^{2} b^{\varphi}+E_{0} U_{\mathrm{AC}}^{\varphi} b^{\varphi} \cos \left(\delta_{0}^{\varphi}-\delta_{\mathrm{AC}}^{\varphi}\right) \\
& -\sum_{\varphi \in\{\mathrm{a}, \mathrm{b}, \mathrm{c}\}} E_{0} U_{\mathrm{AC}}^{\varphi} g^{\varphi} \sin \left(\delta_{0}^{\varphi}-\delta_{\mathrm{AC}}^{\varphi}\right)
\end{aligned}
$$

where $\delta_{0}^{a}=\delta_{0}, \quad \delta_{0}^{b}=\delta_{0}-120^{\circ}, \quad$ and $\quad \delta_{0}^{c}=\delta_{0}+120^{\circ}$. $g=r /\left(r^{2}+x^{2}\right)$, and $b=-x /\left(r^{2}+x^{2}\right)$.

Analogous to the pseudo-measurements for unmonitored loads, a relatively small SE weight should be set for the pseudo-measurements $z_{\mathrm{DG}}$ with a wide range of uncertainty.

\subsubsection{DGs connected to ADNs through PWM}

DGs, connecting to ADNs through PWM converters, operate in different modes depending on the control strategies of PWM converters. The PWM converters link the AC side with the DC side and can control the states of both the $\mathrm{DC}$ and $\mathrm{AC}$ side. It is worth mentioning that control of individual phase outputs is not possible. Instead, the total of three-phase outputs are much more easily controllable in practice [18, 19]. In this paper, the controlled states of DGs are set as pseudo-measurements, and two common control strategies are considered:

1) $P-Q$ mode

When DGs (e.g. storages and fuel cells) operate under this mode, the three-phase total active and reactive power 
$P_{\text {in }}, Q_{\text {in }}$ injected into the $\mathrm{AC}$ network are specified. Accordingly, $z_{\mathrm{DG}}$ can be developed as:

$z_{\mathrm{DG}}=\left[P_{\text {in }} Q_{\text {in }}\right]$

As with (8) and (9), the following equations relating $z_{\mathrm{DG}}$ with $\boldsymbol{x}_{\mathrm{AC}}$ can be obtained:

$$
\begin{aligned}
P_{\mathrm{in}}= & \sum_{\varphi \in\{\mathrm{a}, \mathrm{b}, \mathrm{c}\}}-\left(U_{\mathrm{AC}}^{\varphi}\right)^{2} g^{\varphi}+U_{\mathrm{AC}}^{\varphi} E_{0} g^{\varphi} \cos \left(\delta_{\mathrm{AC}}^{\varphi}-\delta_{0}^{\varphi}\right) \\
& +\sum_{\varphi \in\{\mathrm{a}, \mathrm{b}, \mathrm{c}\}} U_{\mathrm{AC}}^{\varphi} E_{0} b^{\varphi} \sin \left(\delta_{\mathrm{AC}}^{\varphi}-\delta_{0}^{\varphi}\right) \\
Q_{\mathrm{in}}= & \sum_{\varphi \in\{\mathrm{a}, \mathrm{b}, \mathrm{c}\}}\left(U_{\mathrm{AC}}^{\varphi}\right)^{2} b^{\varphi}-U_{\mathrm{AC}}^{\varphi} E_{0} b^{\varphi} \cos \left(\delta_{\mathrm{AC}}^{\varphi}-\delta_{0}^{\varphi}\right) \\
& +\sum_{\varphi \in\{\mathrm{a}, \mathrm{b}, \mathrm{c}\}} U_{\mathrm{AC}}^{\varphi} E_{0} g^{\varphi} \sin \left(\delta_{\mathrm{AC}}^{\varphi}-\delta_{0}^{\varphi}\right)
\end{aligned}
$$

\section{2) $U_{\mathrm{DC}}-Q$ mode}

Under this control strategy, the voltage $U_{\mathrm{DC}}$ of PWM converters on the DC side and the reactive power $Q_{\text {in }}$ injected into the AC network are set as given values. As $U_{\mathrm{DC}}$ cannot be treated as measurements for AC networks straightforwardly, the equality constraints of the DC and $\mathrm{AC}$ side of PWM converters are given as:

$P_{0}=P_{\mathrm{DC}}=f\left(U_{\mathrm{DC}}\right)$

$E_{0}=k_{0} M_{0} U_{\mathrm{DC}}$

where $k_{0}=\sqrt{3} /(2 \sqrt{2})$, and $f$ represents the power-voltage nonlinear model on the DC side which can be understood as $\mathrm{DC}$ power flow (PF) equations.

In DC transmission networks, it needs an iterative solution of PF or SE as in AC networks. A DC network with DGs and PWM converters is relatively simple; the DC power $P_{\mathrm{DC}}$ of the PWM converter on the DC side is independent of the states on the $\mathrm{AC}$ side and can be derived without any iterations. Thereby, one can get $P_{0}$ from $U_{\mathrm{DC}}$ based on (13), and $z_{\mathrm{DG}}$ can be developed as:

$z_{\mathrm{DG}}=\left[\begin{array}{ll}P_{0} & Q_{\mathrm{in}}\end{array}\right]$

In this case the mathematical formula between $\boldsymbol{z}_{\mathrm{DG}}$ and $\boldsymbol{x}_{\mathrm{AC}}$ can be referred to (8) and (12).

The actual states of the DGs controlled by PWM are very close to their given values. Given this, the accuracy of the pseudo-measurements $z_{\mathrm{DG}}$ obtained from (10) or (15) is high.

For each DG connecting to ADNs, a pair of new variables $\left(E_{0}\right.$ and $\left.\delta_{0}\right)$ is added into state variables. Correspondingly, based on (7), (10), or (15), two pseudomeasurements $z_{\mathrm{DG}}$, are introduced. Thereby, the following relationship between new variables and pseudo-measurements for DGs is given by:

$\operatorname{dim}\left(z_{\mathrm{DG}}\right)=\operatorname{dim}\left(\left[\begin{array}{ll}E_{0} & \delta_{0}\end{array}\right]\right)$

If there are additionally real-time measurements for DGs, it will produce a redundancy for DGs. Thus, by means of setting pseudo-measurements for DGs as illustrated above, the ADN integrated with DGs is observable.

In addition, for the DGs connected to ADNs through PWM converters, after the convergence of WLS-based ADNSE, the states of PWM converters $\left(M_{0}\right)$ and the states on the DC side $\left(P_{\mathrm{DC}}\right.$ and $\left.U_{\mathrm{DC}}\right)$ can be calculated from the known $E_{0}$ and $P_{0}$ based on (13)-(14).

\section{Multi-area DSE for distribution networks}

A fully distributed state estimator for multi-area distribution networks is described in this section. In a DSE scheme, each local subarea performs its own SE and communicates with neighboring areas for the exchange of border bus information. The proposed DSE method is on the premise of the following assumptions

1) The subarea division of the whole distribution networks is based on the geographical or topological criteria; each subarea can contain multiple DGs.

2) Neighboring areas share at least one bus and can exchange some amounts of border bus information.

3) Each subarea has an independent control center, e.g. distribution system operators or virtual power plants, to be responsible for carrying out SE and communicating with neighboring areas.

\subsection{Extended areas of distribution networks}

For simplicity, the decomposition of two adjacent areas $K$ and $L$ is illustrated as shown in Fig. 3. In Fig. 3, original areas $K$ and $L$ are two neighboring subareas; buses 1 and 2 are the boundary buses and belong to areas $K$ and $L$, respectively. After decomposition, the original interconnected areas $K$ and $L$ are decomposed into extended areas $K$ and $L$ including all external boundary buses (bus 1 or bus 2). As established on the copies of boundary buses, this achieves area decoupling.

As the measurements in ADNs are rather limited, the designed DSE scheme should take full advantage of boundary bus and line measurements. Fortunately, it is easy to deal with the boundary measurements for the proposed network decomposition scheme for which all boundary buses are included in the extended areas. The conventional boundary measurement processing method 


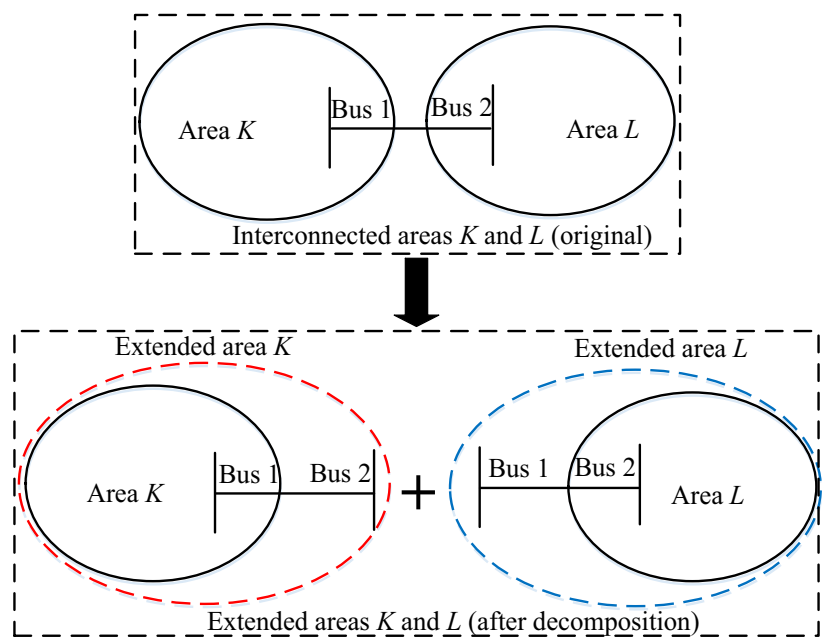

Fig. 3 Concept of extended areas

[23] that the related boundary topology, network parameters and local SE results to the central coordinator can be initially avoided.

Let $\boldsymbol{x}_{K}[L]$ and $\boldsymbol{x}_{L}[K]$ be the boundary sub-variables of the extended areas $K$ and $L$. For example, in Fig. 3, $\boldsymbol{x}_{K}[L]$ (or $\boldsymbol{x}_{L}[K]$ ) consists of the three-phase bus voltages of $\{1,2\}$. For a valid solution, neighboring areas must consent on the boundary sub-variables:

$\boldsymbol{x}_{K}[L]=\boldsymbol{x}_{L}[K]$

Next, the SE problem between neighboring subareas $\mathrm{K}$ and $L$ is given as follows:

$\min _{\left(\mathbf{x}_{K}, \mathbf{x}_{L}\right)}\left\{J_{K}\left(\boldsymbol{x}_{K}\right)+J_{L}\left(\boldsymbol{x}_{L}\right) \mid \boldsymbol{x}_{K}[L]=\boldsymbol{x}_{L}[K]\right\}$

where $\boldsymbol{x}_{K}$ and $\boldsymbol{x}_{L}$ are the augmented state variables for the extended areas $K$ and $L$, respectively.

\subsection{Augmented Lagrangian relaxation}

To solve the optimization problem (18), the equality constraint $\boldsymbol{x}_{K}[L]=\boldsymbol{x}_{L}[K]$ needs to be dualized. Moreover, the augmented Lagrangian relaxation [26, 33] with a quadratic penalty term is introduced to improve the convergence property. As a result, the augmented Lagrangian function for (18) becomes

$\min _{\left(\boldsymbol{x}_{K}, \boldsymbol{x}_{L}, \lambda, \alpha\right)}\left\{\begin{array}{r}J_{K}\left(\boldsymbol{x}_{K}\right)+J_{L}\left(\boldsymbol{x}_{L}\right)+\lambda^{\mathrm{T}}\left(\boldsymbol{x}_{K}[L]-\boldsymbol{x}_{L}[K]\right) \\ +\alpha|| \boldsymbol{x}_{K}[L]-\boldsymbol{x}_{L}[K] \|_{2}^{2}\end{array}\right\}$

where $\lambda$ is the Lagrangian multiplier vector, and $\alpha$ is a positive penalty factor.

The problem (19) cannot be decomposed directly because the same augmented Lagrangian function couples the SE tasks across areas $K$ and $L$. To enable a fully distributed scheme, the block coordinate descent (BCD) method is adopted. This will yield two separate sub-problems (20)-(21) and a multiplier update (22) as:

$\boldsymbol{x}_{K}^{(t+1)}=\arg \min _{\boldsymbol{x}_{K}}\left\{\begin{aligned} J_{K}\left(\boldsymbol{x}_{K}\right)+ & \left(\boldsymbol{\lambda}^{(t)}\right)^{\mathrm{T}}\left(\boldsymbol{x}_{K}[L]-\boldsymbol{x}_{L}[K]^{(t)}\right) \\ & +\frac{\alpha^{(t)}}{2}\left\|\boldsymbol{x}_{K}[L]-\boldsymbol{x}_{L}[K]^{(t)}\right\|_{2}^{2}\end{aligned}\right\}$

$\boldsymbol{x}_{L}^{(t+1)}=\arg \min _{\boldsymbol{x}_{L}}\left\{\begin{array}{r}J_{L}\left(\boldsymbol{x}_{L}\right) \\ +\left(\boldsymbol{\lambda}^{(t)}\right)^{\mathrm{T}}\left(\boldsymbol{x}_{K}[L]^{(t+1)}-\boldsymbol{x}_{L}[K]\right) \\ +\frac{\alpha^{(t)}}{2}\left\|\boldsymbol{x}_{K}[L]^{(t+1)}-\boldsymbol{x}_{L}[K]\right\|_{2}^{2}\end{array}\right\}$

$\lambda^{(t+1)}=\lambda^{(t)}+\eta^{(t)}\left(\boldsymbol{x}_{K}[L]^{(t+1)}-\boldsymbol{x}_{L}[K]^{(t+1)}\right)$

where $t$ represents the iteration number; and $\eta^{(t)}$ is a step size parameter.

Note that the augmented Lagrangian relaxation here severs as a local algorithm as $J_{K}\left(\boldsymbol{x}_{K}\right)$ and $J_{L}\left(\boldsymbol{x}_{L}\right)$ are nonconvex (nonlinear power flow equations). Based on the distributed procedure (20)-(22), neighboring subareas $K$ and $L$ solve (20) and (21) independently. Meanwhile, only a very limited boundary information $\left(x_{K}[L]^{(t)}\right.$ or $\left.x_{L}[K]^{(t)}\right)$ needs to be exchanged between subareas $K$ and $L$ at each successive iteration. The exchanges can be implemented in a fully distributed architecture.

\subsection{Pseudo-measurements for extended boundary buses}

To narrow the gap between the proposed DSE scheme (20) or (21) and the ISE scheme (1), the DSE problem (20) for subarea $K$ can be rewritten as follows (the constant term is neglected)

$\boldsymbol{x}_{K}^{(t+1)}=\arg \min _{\boldsymbol{x}_{K}}\left\{J_{K}\left(\boldsymbol{x}_{K}\right)+\frac{\alpha^{(t)}}{2}\left\|z_{K}^{\mathrm{pse}}-\boldsymbol{x}_{K}[L]\right\|_{2}^{2}\right\}$

$z_{K}^{\mathrm{pse}}=\boldsymbol{x}_{L}[K]^{(t)}-\frac{1}{\alpha^{(t)}} \lambda^{(t)}$

where $z_{K}^{\text {pse }}$ is the introduced measurement vector for extended area $K$.

Comparing the WLS formulation (1) with the second term of (23), it is noted that $z_{K}^{\text {pse }}$ can be understood as the equivalent pseudo-measurements for extended subareas $K$. Here the pseudo-measurements $z_{K}^{\mathrm{pse}}$ whose SE weight is $\alpha / 2$ have a linear relationship with $\boldsymbol{x}_{K}[L]$.

Similarly, the pseudo-measurements $z_{L}^{\text {pse }}$ for extended subareas $K$ corresponding to (21) can be defined as

$z_{L}^{\mathrm{pse}}=\boldsymbol{x}_{K}[L]^{(t+1)}+\frac{1}{\alpha^{(t)}} \lambda^{(t)}$ 
Table 1 Control strategies and pseudo-measurements of DGs

\begin{tabular}{lllll}
\hline DG No. & DGs connected to ADS & Control strategies & \multicolumn{2}{l}{ Pseudo-measurements for DGs } \\
\cline { 3 - 5 } & & & $P(\mathrm{~kW})$ & $P_{0}=600$ \\
\hline DG1 & Directly & - & $P_{0}=550$ & $Q_{0}=197.2$ \\
DG2 & Through PWM & $U_{\mathrm{DC}}-Q$ & $P_{\text {in }}=650$ & $Q_{\text {in }}=137.8$ \\
DG3 & Through PWM & $P-Q$ & $P_{0}=700$ & $Q_{\text {in }}=213.7$ \\
DG4 & Through PWM & $U_{\text {DC }}-Q$ & $P_{\text {in }}=400$ & $Q_{\text {in }}=230.1$ \\
DG5 & Through PWM & $P-Q$ & & $Q_{\text {in }}=131.5$ \\
\hline
\end{tabular}

Under the DSE scheme illustrated above, the dimension of the pseudo-measurements and the boundary state variables for extended subareas is always equal

$\operatorname{dim}\left(z_{K}^{\mathrm{pse}}\right)=\operatorname{dim}\left(\boldsymbol{x}_{K}[L]\right)$ or $\operatorname{dim}\left(z_{L}^{\mathrm{pse}}\right)=\operatorname{dim}\left(\boldsymbol{x}_{L}[K]\right)$

Thus, for each pair of neighboring areas $K$ and $L$, the proposed DSE scheme will keep the observability of the system. There is no further requirement for boundary measurement redundancy as long as local SEs or ISEs are observable. The proposed multi-area DSE procedure is detailed in Algorithm 1. Notice that the penalty factor $\alpha$ is updated by $\alpha^{(t)}=\gamma \alpha^{(t-1)}$ to accelerate convergence.

The distribution network could contain multiple adjacent areas besides areas $K$ and $L$. The proposed DSE is also suitable for other pair of interconnected areas.

By interpreting exchanged information as equivalent pseudo-measurements, the DSE scheme keeps the WLS formulation for each area. As such, for distributed solutions, the bad data detection and identification can be performed the same as integrated solutions. If the measurement redundancy is enough, robust state estimators $[8,34,35]$ may be further implemented.

\section{Case studies}

This section presents test results corresponding to the modified IEEE123-bus system. The data on the IEEE 123bus system was obtained from [36]. Five DGs are connected to buses $1,76,100,95$ and 42 , respectively. The control strategies and pseudo-measurements for DGs are shown in Table 1. For DG1, $r_{\varphi}$ and $x_{\varphi}$ are set as 0.02 per unit. For DG2-DG5, $r_{\varphi}$ and $x_{\varphi}$ are set as 0.02 per unit and 0.2 per unit, respectively. Measurement data with Gaussian noise was generated from the exact power flow results. The maximum percentage measurement errors have been assumed as: $1.5 \%$ (bus voltage and branch current magnitude measurements), 3\% (power flow measurements) and $50 \%$ (pseudo-measurements for loads and DGs connecting to ADNs directly). The virtual measurements for zero injections and pseudo-measurements for DGs connected to

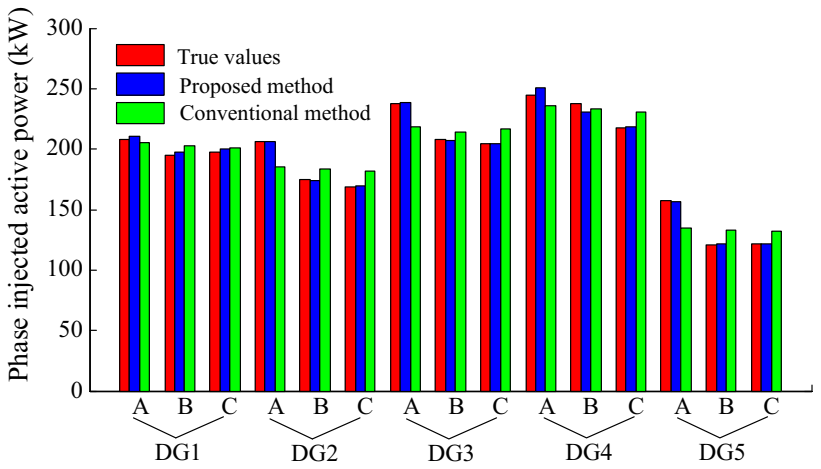

Fig. 4 DG phase injected power comparison between conventional method and proposed method

ADNs through PWM converters have a very low variance $\left(10^{-6}\right)$. For a given maximum error $e_{z i}$ about the mean value $\mu_{z i}$, the standard of deviation $\sigma_{z i}$ of the measurements errors can be computed by

$\sigma_{z i}=\frac{\mu_{z i} \times e_{z i}}{3 \times 100}$

The simulation was executed on a 32-bit PC with 1.9$\mathrm{GHz}$ CPU and 4.0-GB RAM in MATLAB environment.

\subsection{The effectiveness of proposed DG models}

To demonstrate the performance of the proposed DG models, errors in SE results by using the proposed method (considering the asymmetric characteristics of DG threephase outputs) and the conventional method (assuming the phase outputs of DGs in balanced form) are presented in Fig. 4 and Table 2 .

It can be seen from Fig. 4 and Table 2 that the estimation accuracy by using the proposed method is generally better. Because the modified IEEE 123-bus system operates under unbalanced conditions, the true three-phase outputs of DGs are not in balanced form as shown in Fig. 4. Consequently, the phase-based averaging assumption from the conventional method fails to reveal the unbalanced operating states of DGs, leading to a relatively low 
Table 2 Estimation errors for two methods with respect to true values

\begin{tabular}{lll}
\hline Method & Maximum absolute voltage error & Mean absolute voltage error \\
\hline Proposed method & $1.9 \times 10^{-3}$ & $4.2 \times 10^{-4}$ \\
Conventional method & $5.4 \times 10^{-3}$ & $9.7 \times 10^{-4}$ \\
\hline
\end{tabular}

estimation accuracy of DG three-phase outputs. Furthermore, the inappropriate DG models used by the conventional method introduce an additional error (or can be understood as corrupted power injection measurements) to the SE calculation, hence the overall estimation accuracy of the whole network states decreases as shown in Table II. In comparison, attributed to the detailed modelling of unbalanced DG power outputs, SE results with a higher quality are available in the proposed method.

Notice that the accuracy of SE results presented in Fig. 4 and Table 2 is acceptable for engineering applications. But it is still possible that high-quality SE results are unavailable considering the limited redundancy of realtime measurements and the pseudo-measurements with low accuracy. In this case, two promising solutions could be taken to enhance the SE performance: (1) data-driven state estimation [37], in which informative historical data is further taken advantage of to exploit its connection with the current states, and (2) (optimal) placement of PMU units that can effectively increase the SE accuracy (even though the number of PMUs is limited).

\subsection{Performance of DSE in a multi-area framework}

A set of test systems with an increased number of areas was designed by replicating the modified IEEE 123-bus system to investigate the numerical efficiency of the proposed DSE method. Three different examples are described in Fig. 5:

EX1 (four areas): three replicated areas are connected to buses 13, 28 and 64;

EX2 (six areas): on the basis of EX1, another two replicated areas are connected buses 21 and 105;

EX3 (eight areas): on the basis of EX2, another two replicated areas are connected buses 57 and 135.

Figure 6 presents the computational efficiency comparison between centralized and distributed schemes with respect to EX1-EX3. With the increase of interconnected areas (or bus numbers), the computational burden of the centralized scheme is much heavier than the proposed distributed scheme. This is attributed to the fact that when more areas are replicated, for the distributed scheme, the computational complexity of SE in each area is relatively constant, and only a bit more communication time (or more iteration numbers for local SEs) is needed. But for the

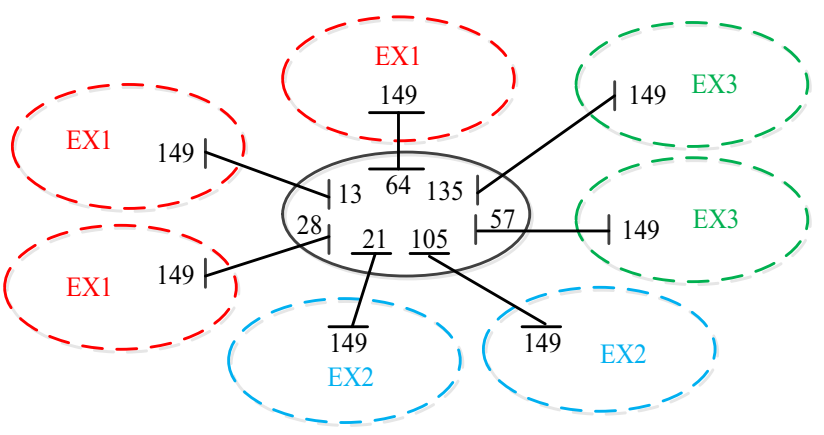

Fig. 5 Diagram of the interconnected multi-area systems by replicating IEEE 123-bus system

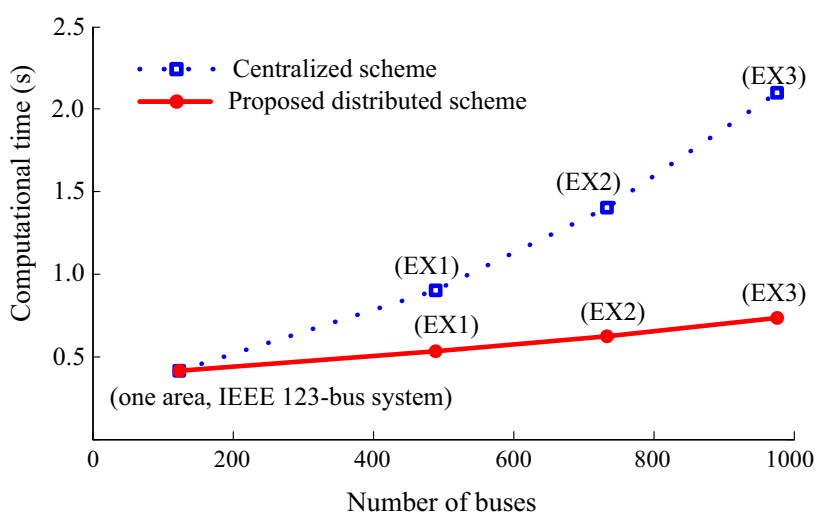

Fig. 6 Computational efficiency comparison between centralized and distributed scheme with respect to EX1-3

centralized scheme, the SE computational complexity is increased with a larger network scale.

On the other hand, numerical stability is another difficult issue faced by SEs at the distribution levels. The numerical stability comparison between the centralized and distributed schemes with respect to EX1-EX3 is depicted in Fig. 7. The condition number of the gain matrix is a key indicator concerning the numerical stability of SE [38]. A relatively high condition number may result in an unstable numerical solution. As seen in Fig. 7, the distributed scheme overshadows the centralized scheme in terms of the numerical stability. As a result of the reduced scale of the SE problem, the numerical stability of the distributed scheme is thereby enhanced.

To conclude, the tests regarding computational time and numerical stability corroborate that the computational and numerical efficiency of the proposed DSE algorithm scale 


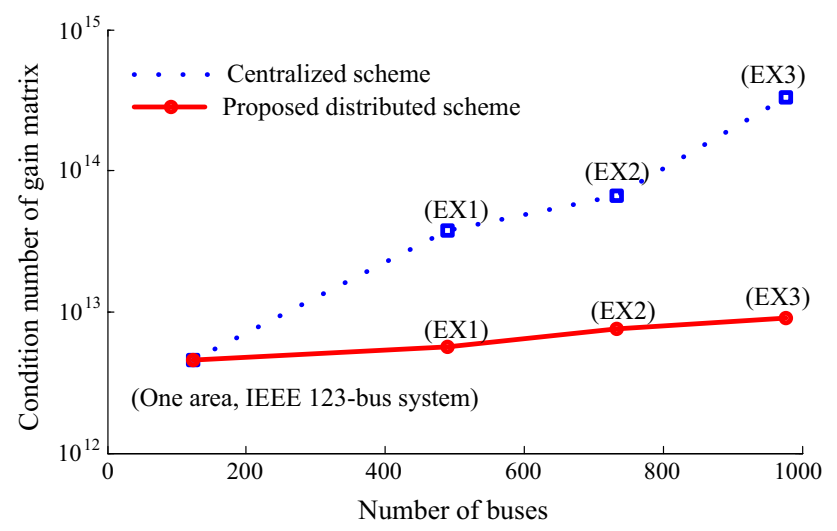

Fig. 7 Numerical stability comparison between centralized and distributed scheme with respect to EX1-3

are favorable with the interconnected distribution network size.

The stopping criterion was set as 0.001 per unit. Accordingly, for the DSE scheme, three examples reached convergence with iteration numbers being 7,9 and 12, respectively. Meanwhile, the voltage difference between DSE and ISE was less than $0.1 \%$. Thus, the convergence property and the precision (or optimality) of the augmented Lagrangian-based DSE were generally satisfactory. It is also relevant to clarify that there exists a compromise between precision and computational complexity for DSE. The higher precision of the stopping criterion, the more computational time is needed.

\section{Conclusions}

A scalable and efficient state estimator that can address the two main challenges (unbalanced modeling of DGs and computational efficiency issues) of ADNSE has been proposed in this paper. A steady-state three-phase DG model considering the unbalanced characteristics of DG threephase outputs was initially proposed. Subsequently, a feasible strategy to set pseudo-measurements for DGs has been presented. Furthermore, on the premise of extended areas, the augmented Lagrangian-based DSE method was specially designed for multi-area ADNs. As evidenced by the test results, three aspects of advantages for the proposal in this work have been observed: higher accuracy, lower computational effort and superior numerical stability. The proposed DG model attends the first advantage, and the last two are owed to the fully distributed framework. Therefore, this novelty method proposed in our work is a strong stimulus for the smart distribution system implementations.

Emerging future research direction will focus on this work's application to the ADN operating interdependently with multi-microgrids and the AC-DC hybrid distribution networks (or microgrids).

Acknowledgment This work was supported by National Natural Science Foundation of China (No. 51277052).

Open Access This article is distributed under the terms of the Creative Commons Attribution 4.0 International License (http:// creativecommons.org/licenses/by/4.0/), which permits unrestricted use, distribution, and reproduction in any medium, provided you give appropriate credit to the original author(s) and the source, provide a link to the Creative Commons license, and indicate if changes were made.

\section{References}

[1] Schweppe FC, Wildes J (1970) Power system static-state estimation, Part I: exact model. IEEE Trans Power Appar Syst 89(1):120-125

[2] Abur A, Exposito AP (2004) Electric power system state estimation. Marcel Dekker, New York

[3] Wang D, Ge SY, Jia HJ et al (2014) A demand response and battery storage coordination algorithm for providing microgrid tie-line smoothing services. IEEE Trans Sustain Energy 5(2):476-486

[4] Lu CN, Ten JH, Liu WHE (1995) Distribution system state estimation. IEEE Trans Power Syst 10(1):229-240

[5] Baran ME, Kelley AW (1995) A branch-current-based state estimation method for distribution systems. IEEE Trans Power Syst 10(1):483-491

[6] Wang HB, Schulz NN (2004) A revised branch current-based distribution system state estimation algorithm and meter placement impact. IEEE Trans Power Syst 19(1):207-213

[7] Pau M, Pegoraro PA, Sulis S (2013) Efficient branch-currentbased distribution system state estimation including synchronized measurements. IEEE Trans Instrum Meas 62(9):2419-2429

[8] Göl M, Abur A (2014) A robust PMU based three-phase state estimator using model decoupling. IEEE Trans Power Syst 29(5):2292-2299

[9] Wu JZ, He Y, Jenkins N (2013) A robust state estimator for medium voltage distribution networks. IEEE Trans Power Syst 28(2):1008-1016

[10] Singh R, Pal BC, Jabr RA (2009) Choice of estimator for distribution system state estimation. IET Gener Transm Distrib 3(7):666-678

[11] Singh R, Pal BC, Jabr RA (2010) Distribution system state estimation through Gaussian mixture model of the load as pseudo-measurement. IET Gener Transm Distrib 4(1):50-59

[12] Muscas C, Pilo F, Pisano G et al (2009) Optimal allocation of multichannel measurement devices for distribution state estimation. IEEE Trans Instrum Meas 58(6):1929-1937

[13] Liu JQ, Ponci F, Monti A et al (2014) Optimal meter placement for robust measurement systems in active distribution grids. IEEE Trans Instrum Meas 63(5):1096-1105

[14] Wei ZN, Yang X, Sun GQ et al (2013) Wind power system state estimation with automatic differentiation technique. Int J Electr Power Energy Syst 53:297-306

[15] Haughton DA, Heydt GT (2013) A linear state estimation formulation for smart distribution systems. IEEE Trans Power Syst 28(2):1187-1195 
[16] Ranković A, Maksimović BM, Sarić AT et al (2014) A threephase state estimation in active distribution networks. Int $\mathbf{J}$ Electr Power Energy Syst 54(1):154-162

[17] Ranković A, Maksimović BM, Sarić AT et al (2015) ANNbased correlation of measurements in micro-grid state estimation. Int Trans Electr Energy Syst 25(10):2181-2202

[18] Hwang PI, Jang G, Moon SI et al (2016) Three-phase steadystate models for a distributed generator interfaced via a currentcontrolled voltage-source converter. IEEE Trans Smart Grid 7(3):1694-1702

[19] Abdelaziz MMA, Farag HE, El-Saadany EF et al (2013) A novel and generalized three-phase power flow algorithm for islanded microgrids using a Newton trust region method. IEEE Trans Power Syst 28(1):190-201

[20] Giustina DD, Pau M, Pegoraro PA et al (2014) Electrical distribution system state estimation: measurement issues and challenges. IEEE Instrum Meas Mag 17(6):36-42

[21] Klauber C, Zhu H (2015) Distribution system state estimation using semidefinite programming. In: Proceedings of the 2015 North American power symposium (NAPS'15), Charlotte, NC, USA, 4-6 Oct 2015, 6 pp

[22] Gómez-Expósito A, de la Villa Jaén A, Gómez-Quiles C et al (2011) A taxonomy of multi-area state estimation methods. Electr Power Syst Res 81(4):1060-1069

[23] Zhao L, Abur A (2005) Multi area state estimation using synchronized phasor measurements. IEEE Trans Power Syst 20(2):611-617

[24] Zhu H, Giannakis GB (2014) Power system nonlinear state estimation using distributed semidefinite programming. IEEE J Sel Top Signal Process 8(6):1039-1050

[25] Minot A, Lu YM, Li N (2015) A distributed gauss-newton method for power system state estimation. IEEE Trans Power Syst. doi:10.1109/TPWRS.2015.2497330

[26] Kekatos V, Giannakis GB (2013) Distributed robust power system state estimation. IEEE Trans Power Syst 28(2):1617-1626

[27] Xie L, Choi DH, Kar S et al (2012) Fully distributed state estimation for wide-area monitoring systems. IEEE Trans Smart Grid 3(3):1154-1169

[28] Muscas C, Pau M, Pegoraro PA et al (2015) Multiarea distribution system state estimation. IEEE Trans Instrum Meas 64(5):1140-1148

[29] Nordman MM, Lehtonen M (2005) Distributed agent-based state estimation for electrical distribution networks. IEEE Trans Power Syst 20(2):652-658

[30] Nguyen PH, Kling WL, Myrzik JMA (2009) Completely decentralized state estimation for active distribution network. In: Proceedings of the 9th IASTED European conference, Palma de Mallorca, Spain, 7-9 Sept 2009, 6 pp

[31] Alimardani A, Zadkhast S, Jatskevich J, et al (2014) Using smart meters in state estimation of distribution networks. In: Proceedings of the 2014 IEEE Power and Energy Society general meeting, National Harbor, MD, USA, 27-31 Jul 2014, 5 pp

[32] Kamh MZ, Iravani R (2012) Steady-state model and power-flow analysis of single-phase electronically coupled distributed energy resources. IEEE Trans Power Deliv 27(1):131-139

[33] Liu C, Shahidehpour M, Wang JH (2010) Application of augmented Lagrangian relaxation to coordinated scheduling of interdependent hydrothermal power and natural gas systems. IET Gener Transm Distrib 4(12):1314-1325

[34] Chen YB, Ma J (2014) A mixed-integer linear programming approach for robust state estimation. J Mod Power Syst Clean Energy 2(4):366-373. doi:10.1007/s40565-014-0078-7

[35] Wu WC, Ju YT, Zhang BM et al (2012) A distribution system state estimator accommodating large number of ampere measurements. Int J Electr Power Energy Syst 43(1):839-848
[36] IEEE, PES Distribution System Analysis Subcommittee's Distribution Test Feeder Working Group (2012) Distribution test feeder. IEEE Power and Energy Society, Piscataway

[37] Weng Y, Negi R, Ilić MD (2013) Historical data-driven state estimation for electric power systems. In: Proceedings of the 2013 IEEE international conference on Smart Grid Communications (SmartGridComm'13), Vancouver, Canada, 21-24 Oct 2013, 6 pp

[38] Dasgupta K, Swarup KS (2011) Tie-line constrained distributed state estimation. Int J Electr Power Energy Syst 33(3):569-576

Sheng CHEN received his Bachelor degree from the College of Energy and Electrical Engineering, Hohai University, Nanjing, China, in 2014, where he is currently pursuing the Ph.D. degree. His research interests include economic dispatch and security analysis of integrated energy systems.

Zhinong WEI received the B.S. degree from Hefei University of Technology, Hefei, China, in 1984, the M.S. degree from Southeast University, Nanjing, China, in 1987, and the Ph.D. degree from Hohai University, Nanjing, China, in 2004. He is now a Professor of electrical engineering with the College of Energy and Electrical Engineering, Hohai University, Nanjing, China. His research interests include power system state estimation, integrated energy systems, smart distribution systems, optimization and planning, load forecasting, and integration of distributed generation into electric power systems.

Guoqiang SUN received the B.S., M.S., and Ph.D. degrees in electrical engineering from Hohai University, Nanjing, China, in 2001, 2005, and 2010, respectively. He was a visiting scholar at North Carolina State University, Raleigh, NC from 2015 to 2016. He is now an Associate Professor with the College of Energy and Electrical Engineering, Hohai University, Nanjing, China. His research interests include power system analysis and economic dispatch and optimal control of integrated energy systems.

Ning $\mathbf{L} \mathbf{U}$ received the B.Sc. degree in electrical engineering from the Harbin Institute of Technology, Harbin, China, in 1993, and the M.Sc. and $\mathrm{Ph} . \mathrm{D}$. degrees in electric power engineering from Rensselaer Polytechnic Institute, Troy, NY, USA, in 1999 and 2002, respectively. She is an Associate Professor with the Department of Electrical and Computer Engineering, North Carolina State University, Raleigh, NC, USA.

Yonghui SUN received the M.S. degree in applied mathematics from Southeast University, Nanjing, China, in 2007, and the Ph.D. degree in control theory and application from City University of Hong Kong, Hong Kong, in 2010. He is now a Professor of electrical engineering with the College of Energy and Electrical Engineering, Hohai University, Nanjing, China. $\mathrm{He}$ is an active reviewer for many international journals. His research interests include analysis and control of power systems, stochastic control, complex networks, systems biology, and fuzzy modeling and control.

Ying ZHU received the B.Sc. and Ph.D. degrees in electrical engineering from the Southeast University, Nanjing, China, in 2008 and 2014, respectively. She is now a lecturer with the College of Energy and Electrical Engineering, Hohai University, Nanjing, China. Her current research interests include application of wind power generation system, novel permanent-magnet machine control, and distribution system analysis. 